\title{
Induced Representations and Hypergroupoids ${ }^{\star}$
}

Jean RENAULT

Université d'Orléans et CNRS (UMR 7349 et FR2964), Département de Mathématiques, F-45067 Orléans Cedex 2, France

E-mail: Jean.Renault@univ-orleans.fr

URL: http://www.univ-orleans.fr/mapmo/membres/renault/

Received February 01, 2014, in final form May 26, 2014; Published online June 03, 2014

http://dx.doi.org/10.3842/SIGMA.2014.057

\begin{abstract}
We review various notions of correspondences for locally compact groupoids with Haar systems, in particular a recent definition due to R.D. Holkar. We give the construction of the representations induced by such a correspondence. Finally, we extend the construction of induced representations to hypergroupoids.
\end{abstract}

Key words: groupoids; $C^{*}$-algebras; correspondences; induced representations; hypergroups

2010 Mathematics Subject Classification: 22D30; 22D25; 22A22; 46L08

Dedicated to Professor Marc A. Rieffel on the occasion of his 75th birthday

\section{Introduction}

Among many great accomplishments, M. Rieffel is well known for the theory of strong Morita equivalence of $C^{*}$-algebras he introduced in his influential article [26] on induced representations for $C^{*}$-algebras. He develops there a $C^{*}$-algebraic setting which generalizes much of G. Mackey's theory of induced representations for locally compact groups. As described in [27], transformation group $C^{*}$-algebras provide many examples of strongly Morita equivalent $C^{*}$-algebras. The notion of groupoid equivalence introduced in $[22,23]$ to give a common framework to these examples is directly inspired by Rieffel's theory. The key fact (stated in [23, Section 3] and proved in [18, Theorem 2.8]) is that a groupoid equivalence implements a strong Morita equivalence of the groupoid $C^{*}$-algebras. However, concerning induced representations, M. Rieffel points out that [26] does not cover some parts of Mackey's theory such as the subgroup theorem or the intertwining number theorem. This raises the question to find a proper setting for these theorems. For that purpose, it seems that a necessary step is to find an analogue at the groupoid level of the notion of $C^{*}$-correspondence. This $C^{*}$-algebraic notion of correspondence appears naturally in [26, Definition 4.19] under the name of Hermitian $B$-rigged $A$-module (we shall simply say $(A, B)-C^{*}$-correspondence, or $C^{*}$-correspondence from $A$ to $B$ ). They (or rather their isomorphism classes) can be viewed as morphisms in a category having $C^{*}$-algebras as objects; Morita equivalences are isomorphisms in this category. Since [26], various notions of correspondences for groupoids have been proposed. They will be reviewed in Section 2 of this article. However, it is only recently that, building upon the previous definitions, a satisfactory notion has been found by R.D. Holkar. It has two big advantages: first, it makes the groupoid $C^{*}$-algebra construction a functor from the category of groupoids to the category of $C^{*}$-algebras and second, it implements many classical cases of induced representations. In Section 3, we shall describe the construction of representations induced through a groupoid correspondence and give some examples which illustrate this notion. In Section 4, we shall see the necessity to enlarge the category of groupoids to capture some natural constructions of induced representations. This will

\footnotetext{
${ }^{\star}$ This paper is a contribution to the Special Issue on Noncommutative Geometry and Quantum Groups in honor of Marc A. Rieffel. The full collection is available at http://www.emis.de/journals/SIGMA/Rieffel.html
} 
lead us to the notion of locally compact hypergroupoid with Haar system and to the construction of their $C^{*}$-algebras. An important class of examples is provided by double coset hypergroups, and more generally by spatial hypergroupoids which have been considered earlier in [11].

The groupoid $C^{*}$-algebra construction works very well for non-Hausdorff groupoids (more precisely topological groupoids such that each point has a compact Hausdorff neighborhood, the unit space is Hausdorff and there is a continuous Haar system). Nevertheless, for the sake of simplicity, we shall assume here that our locally compact spaces and groupoids are Hausdorff and second countable. Most of the theory goes through in the non-Hausdorff case but a systematic treatment would impair the legibility of the exposition. When we consider Borel spaces and groupoids, we implicitly assume that they are analytic.

We use the terminology and the notation of [1]. The unit space of a groupoid $G$ is denoted by $G^{(0)}$. The elements of $G$ are usually denoted by $\gamma, \gamma^{\prime}, \ldots$; those of $G^{(0)}$ are denoted by $x, y, \ldots$ or $u, v \ldots$ The structure of $G$ is defined by the inclusion map $i: G^{(0)} \rightarrow G$ (we shall identify $x$ and $i(x)$ ), the range and source maps $r, s: G \rightarrow G^{(0)}$, the inverse map $\gamma \mapsto \gamma^{-1}$ from $G$ to $G$ and the multiplication map $\left(\gamma, \gamma^{\prime}\right) \mapsto \gamma \gamma^{\prime}$ from the set of composable pairs

$$
G^{(2)}=\left\{\left(\gamma, \gamma^{\prime}\right) \in G \times G: s(\gamma)=r\left(\gamma^{\prime}\right)\right\}
$$

to $G$. Given $A, B \subset G^{(0)}$, we write $G^{A}=r^{-1}(A), G_{B}=s^{-1}(B)$ and $G_{B}^{A}=G^{A} \cap G_{B}$. Similarly, given $x, y \in G^{(0)}$, we write $G^{x}=r^{-1}(x), G_{y}=s^{-1}(y)$ and $G(x)=G_{x}^{x}$. We assume that $G^{(0)} \subset G$ and $G^{(2)} \subset G \times G$ have the subspace topology. We also include in the definition of a topological groupoid the assumptions that the range and source maps are open.

Most spaces occuring in the theory of groupoids are fibered spaces: we shall say that a set $Y$ is fibered over a set $X$ if a surjective map $p: Y \rightarrow X$, called the projection map, has been specified. When two sets $Y$ and $Z$ are fibered over $X$ via the maps $p: Y \rightarrow X$ and $q: Z \rightarrow X$, we define the fibered product

$$
Y * Z=\{(y, z) \in Y \times Z: p(y)=q(z)\} .
$$

It is also a set fibered over $X$. A left $G$-space consists of a set $X$ fibered over $G^{(0)}$ by a map $r_{X}: X \rightarrow G^{(0)}$, called the projection or moment map and a map $(\gamma, x) \in G * X \mapsto \gamma x \in X$, called the action map, where $G$ is fibered over $G^{(0)}$ by the source map, such that the following equalities hold whenever they make sense: $\gamma\left(\gamma^{\prime} x\right)=\left(\gamma \gamma^{\prime}\right) x$ and $u x=x$ for a unit $u \in G^{(0)}$. The relation $x \sim y$ if and only if there exists $\gamma \in G$ such that $x=\gamma y$ is an equivalence relation and the quotient space is denoted by $G \backslash X$. One defines similarly a right $G$-space $X$. Then the moment map is denoted by $s_{X}$ and the quotient space by $X / G$. An $(H, G)$-space, where $H, G$ are groupoids, is a space $X$ which is both a left $H$-space and a right $G$-space and such that $(h x) \gamma=h(x \gamma)$ for all $(h, x, \gamma) \in H * X * G$. In the locally compact setting, we assume that $X$ and $G$ are locally compact, the moment map is continuous and open and the action map is continuous. One says that the left $G$-space $X$ is free if the map from $G * X$ to $X \times X$ sending $(\gamma, x)$ to $(\gamma x, x)$ is one-to-one and that it is proper if this map is proper. One says that the groupoid $G$ is proper if the left $G$-space $G^{(0)}$ is proper. In the Borel setting, we assume that $X$ and $G$ are Borel and that the moment map and the action map are Borel. Given a left $G$ space $X$, one can form the semi-direct product groupoid $G \ltimes X$ (see, e.g., [1, Subsection 2.1.a]): as a set, it is $G * X$ but it is sometimes preferable to write its elements as $(\gamma x, \gamma, x)$ rather than $(\gamma, x) \in G * X$ to let appear the range of the element. Its unit space is $X$ and $i(x)=\left(x, r_{X}(x), x\right)$; $r(\gamma x, \gamma, x)=\gamma x$ and $s(\gamma x, \gamma, x)=x$; its multiplication is given by

$$
\left(\gamma^{\prime} \gamma x, \gamma^{\prime}, \gamma x\right)(\gamma x, \gamma, x)=\left(\gamma^{\prime} \gamma x, \gamma^{\prime} \gamma, x\right)
$$

and its inverse map by $(\gamma x, \gamma, x)^{-1}=\left(x, \gamma^{-1}, \gamma x\right)$. In the locally compact setting, $G \ltimes X$ becomes a locally compact groupoid. One defines similarly the semi-direct product groupoid $X \rtimes G$ for a right $G$-space $X$. 
Given locally compact (resp. Borel) spaces $X, Y$ and a continuous (resp. Borel) projection map $p: Y \rightarrow X$, a continuous (resp. Borel) $p$-system of measures is a family $\alpha=\left(\alpha_{x}\right)_{x \in X}$ such that $\alpha_{x}$ is a Radon [resp. $\sigma$-finite] measure on $p^{-1}(x)$ and such that for all function $f$ on $Y$ complex-valued, continuous with compact support (resp. positive and Borel), $x \mapsto \int f d \alpha_{x}$ is continuous (resp. Borel) on $Y$; this function is denoted $\alpha(f)$. If $X, Y$ are (say right) $G$-spaces and $p$ is equivariant, we say that $\alpha$ is equivariant if for all $\gamma \in G, \alpha_{r(\gamma)} \gamma=\alpha_{s(\gamma)}$. A (left) Haar system for the locally compact groupoid $G$ is a continuous and equivariant $r$-system of measures $\lambda=\left(\lambda^{x}\right)_{x \in G^{(0)}}$ for the left $G$-space $G$. It is an essential piece of data for defining the convolution algebra $C_{c}(G, \lambda)$ and the reduced and full $C^{*}$-algebras $C_{r}^{*}(G, \lambda)$ and $C^{*}(G, \lambda)$ (which are usually denoted simply by $C_{r}^{*}(G)$ and $C^{*}(G)$ ). When $G$ is an étale groupoid, i.e. the range map $r$ is a local homeomorphism, one chooses implicitly the Haar system $\lambda=\left(\lambda^{x}\right)_{x \in G^{(0)}}$ where $\lambda^{x}$ is the counting measure on $G^{x}$.

\section{Groupoid correspondences}

\subsection{Previous definitions}

The notion of groupoid correspondence was introduced at the same time as the notion of groupoid equivalence. In [22], a correspondence from a locally compact groupoid $H$ to another $G$ is defined as an $(H, G)$-space $X$ such that $G$ acts freely and properly. There, the category of locally compact groupoids is obtained by taking isomorphisms classes of correspondences as arrows; isomorphic objects in this category are called equivalent. We shall see that the final definition of a correspondence which will be adopted in this paper is very close to this initial definition: we shall define a correspondence from $H$ to $G$ as an $(H, G)$-space $X$ such that $G$ acts properly (we shall also require some extra data). For consistency throughout the paper, left and right have been exchanged with respect to some original definitions. The reader should keep in mind that a correspondence from $H$ to $G$ should define a $C^{*}$-correspondence from $C^{*}(H)$ to $C^{*}(G)$ and induce representations from $G$ to $H$.

The notion of a Hilsum-Skandalis map, introduced in [9] and emphasized by J. Mrčun in [17] is akin to the above definition. A principal bibundle is an $(H, G)$-space $X$ such that $H$ acts freely and properly and $s_{X}: X \rightarrow G^{(0)}$ identifies $H \backslash X$ and $G^{(0)}$. This is also called a generalized homomorphism since a groupoid homomorphism from $G$ to $H$ defines an $(H, G)$-principal bibundle. A Hilsum-Skandalis map is defined as an isomorphism class of principal bibundles. In the case when $H$ and $G$ are étale and smooth, Mrčun associates to a principal $(H, G)$-bibundle a $\left(C_{c}^{\infty}(H), C_{c}^{\infty}(G)\right)$-bimodule and shows the functoriality of the construction. He also introduces a notion of Morita equivalence for these smooth algebras. These are algebraic constuctions. Let us turn to the $C^{*}$-algebraic framework and recall the now classical notion of $C^{*}$-correspondence.

Definition 2.1. Let $A$ and $B$ be $\mathrm{C}^{*}$-algebras. An $(A, B)-C^{*}$-correspondence is a right $B$ - $\mathrm{C}^{*}$ module $\mathcal{E}$ together with a $*$-homomorphism $\pi: A \rightarrow \mathcal{L}_{B}(\mathcal{E})$.

As is well-known (see [26, Theorem 5.9]), $C^{*}$-correspondences can be composed and strong Morita equivalences are exactly the invertible $C^{*}$-correspondences. The category of $C^{*}$-algebras is defined as the category whose objects are the $C^{*}$-algebras and arrows are the isomorphism classes of $C^{*}$-correspondences. A first step in the generalization of the Muhly-Renault-Williams theorem [18, Theorem 2.8] from equivalences to correspondences was made by M. Macho Stadler and M. O'uchi in [15]. Their definition is equivalent to the following: a correspondence from $H$ to $G$ in the sense of Macho Stadler and O'uchi is an $(H, G)$-space $X$ such that both $G$ and $H$ act properly and $s_{X}: X \rightarrow G^{(0)}$ identifies $H \backslash X$ and $G^{(0)}$. Assuming that $H$ and $G$ have Haar systems, they construct a $C^{*}$-correspondence, which we shall denote by $C_{r}^{*}(X)$, from the reduced 
$C^{*}$-algebra $C_{r}^{*}(H)$ to the reduced $C^{*}$-algebra $C_{r}^{*}(G)$. N. Landsman gives in [14, Section 3.3] the same construction, in the category of Lie groupoids. In his definition of a correspondence from $H$ to $G$, he assumes that the action of $H$ is free and proper and the action of $G$ is proper. He constructs the $C^{*}$-correspondence $C^{*}(X)$ from $C^{*}(H)$ to $C^{*}(G)$ and sketches the proof of the functoriality of this construction. In [29, Theorem 7.11], J.-L. Tu gives the same result, but assuming only that $G$ and $H$ are locally compact, not necessarily Hausdorff, groupoids with Haar systems. He states without proof the functoriality of the construction.

The notion of correspondence used by M. Macho Stadler, M. O'uchi et al. is insufficient. Indeed, it does not cover the restriction of a representation of a locally compact group $G$ to a closed subgroup $H$. In that case $X=G$ as an $(H, G)$-space but the assumption that $H \backslash G=$ $G^{(0)}$ does not hold. Restriction of representations is covered by the following construction. In [4], M. Buneci and P. Stachura define a morphism from $H$ to $G$ as an $(H, G)$-space $X$, where $X$ is $G$ itself and $G$ acts on $X$ by right multiplication. Note that this is a particular case of our initial definition of a correspondence. Assume moreover that $H$ and $G$ are locally compact and have Haar systems. It is not difficult to define an action of $C_{c}(H)$ on $C_{c}(G)$ by multipliers. However, in order to have a $*$-homomorphism from $C^{*}(H)$ into the multiplier algebra $M C^{*}(G)$, it is necessary to introduce a modular function. Since this condition is fairly technical and we are going to give it in a more general framework, we do not reproduce it here. Buneci and Stachura show also that the construction of the $C^{*}$-algebra is functorial, where the arrows in the category of $C^{*}$-algebras are the $*$-homomorphisms $A \rightarrow M B$. One of the drawbacks of their construction is that it does not include groupoid equivalences.

\subsection{Holkar's definition}

Let us now present the notion of correspondence given by R.D. Holkar in his Ph.D. thesis [10], which includes both the correspondences in the sense of Macho Stadler and O'uchi and the morphisms of Buneci and Stachura. We first introduce a notation and a definition.

Let $G$ be a locally compact groupoid and $X$ a locally compact left $G$-space. We form the semi-direct product groupoid $G \ltimes X$. A left Haar system $\lambda$ for $G$ defines $\lambda_{1}$, integration along the fibers of the range map $r: G \ltimes X \rightarrow X$ given by $r(\gamma, x)=\gamma x$ and $\lambda_{2}$, integration along the fibers of the source map $s: G \ltimes X \rightarrow X$, given by $s(\gamma, x)=x$ :

$$
\lambda_{1}(f)(x)=\int f\left(\gamma, \gamma^{-1} x\right) d \lambda^{r(x)}(\gamma), \quad \lambda_{2}(f)(x)=\int f\left(\gamma^{-1}, x\right) d \lambda^{r(x)}(\gamma)
$$

for $f \in C_{c}(G \ltimes X)$.

Definition 2.2. Let $\Delta: G \ltimes X \rightarrow \mathbf{R}_{+}^{*}$ be a Borel cocycle. We say that a measure $\mu$ on $X$ is a $\Delta$-measure with respect to $(G, \lambda)$ if

$$
\mu \circ \lambda_{1}=\Delta\left(\mu \circ \lambda_{2}\right) \text {. }
$$

In other words, $\mu$ is a quasi-invariant measure and admits $\Delta$ as Radon-Nikodym derivative.

Definition $2.3([10])$. Let $(G, \lambda)$ and $(H, \beta)$ be locally compact groupoids with Haar systems. We say that $(X, \alpha)$ is a $\Delta$-correspondence from $(H, \beta)$ to $(G, \lambda)$ if

(i) $X$ is a locally compact space $(H, G)$-space;

(ii) the right action of $G$ is proper;

(iii) $\Delta: H \ltimes(X / G) \rightarrow \mathbf{R}_{+}^{*}$ is a continuous cocycle;

(iv) $\alpha=\left(\alpha_{u}\right)_{u \in G^{(0)}}$ is a continuous $G$-equivariant system of measures for $s_{X}: X \rightarrow G^{(0)}$;

(v) for all $u \in G^{(0)}, \alpha_{u}$ is a $\Delta$-measure with respect to $(H, \beta)$.

We say that $\Delta$ is the module of the correspondence $(X, \alpha)$. 


\subsection{Examples}

a) In the case of a correspondence in the sense of Macho Stadler and O'uchi, one defines the $s_{X}$-system $\alpha$ by

$$
\alpha(f)(u)=\int f\left(h^{-1} x\right) d \beta^{r_{X}(x)}(h),
$$

where $f \in C_{c}(X), u \in G^{(0)}$ and one has chosen $x \in X$ such that $s_{X}(x)=u$; the integral depends only on $u$. In that case $\Delta \equiv 1$. Note that this also includes groupoid equivalences, which are a particular case of Macho Stadler and O'uchi correspondences.

b) In the case of a morphism in the sense of Buneci and Stachura where $X=G$, one defines the $s_{X}$-system $\alpha$ by

$$
\alpha(f)(u)=\int f\left(\gamma^{-1}\right) d \lambda^{u}(\gamma), \quad f \in C_{c}(G) .
$$

Their condition is exactly the assumption (v) of Definition 2.3.

c) Let $H$ be a closed subgroup of a locally compact group $G$ endowed respectively with left Haar measures $\beta$ and $\lambda$. The correspondence which gives induction of a representation of $H$ to a representation of $G$ is $X=G$ as a $(G, H)$-space, endowed with the right Haar measure $\alpha=\lambda^{-1}$. An easy computation shows that $\alpha$ is a 1-measure with respect to $(G, \lambda)$. On the other hand the correspondence which gives the restriction of a representation of $G$ to a representation of $H$ is $Y=G$ as a $(H, G)$-space, again endowed with the right Haar measure $\alpha=\lambda^{-1}$. Another easy computation shows that $\alpha$ is a $\Delta$-measure with respect to $(H, \beta)$, where $\Delta(h)=\delta_{H}(h) / \delta_{G}(h)$ for $h \in H$ and $\delta_{H}, \delta_{G}$ are respectively the modular functions of $H$ and $G$.

\subsection{The $C^{*}$-correspondence}

The construction of the $C^{*}$-correspondence $C^{*}(X, \alpha)$ from a groupoid $(H, G)$-correspondence $(X, \alpha)$ as above is straightforward. We refer to [10] (or to the previous constructions) for details. One first ignores $H$ and constructs the right $C^{*}$-module $\mathcal{E}=C^{*}(X, \alpha)$ over $B=C^{*}(G, \lambda)$ as in [24] or [11]; it is the completion of $C_{c}(X)$ for the following operations: for $f \in C_{c}(G)$ and $\xi, \eta \in C_{c}(X)$,

$$
\xi f(x)=\int \xi(x \gamma) f\left(\gamma^{-1}\right) d \lambda^{s(x)}(\gamma), \quad\langle\xi, \eta\rangle(\gamma)=\int \overline{\xi(x)} \eta(x \gamma) d \alpha_{r(\gamma)}(x) .
$$

The $*$-homomorphism $\pi: C^{*}(H, \beta) \rightarrow \mathcal{L}_{B}(\mathcal{E})$ is defined, for $g \in C_{c}(H)$ and $\xi \in C_{c}(X)$ by

$$
g \xi(x)=\int g(h) \xi\left(h^{-1} x\right) \Delta^{1 / 2}\left(h^{-1}, x\right) d \beta^{r(x)}(h) .
$$

Lemma 2.4. For $g \in C_{c}(H)$ and $\xi, \eta \in C_{c}(X)$, one has $\langle g \xi, \eta\rangle=\left\langle\xi, g^{*} \eta\right\rangle$.

Proof. This is a simple computation which justifies the introduction of the module $\Delta$.

By definition of the full $C^{*}$-norm, $\pi$ extends to $C^{*}(H, \beta)$. This gives the $C^{*}$-correspondence $C^{*}(X, \alpha)$ from $C^{*}(H, \beta)$ to $C^{*}(G, \lambda)$. 


\subsection{Composition of correspondences}

We sketch here the construction of the composition of correspondences. A detailed presentation is given in [10]. The construction leans upon two elementary results which we first recall.

Lemma 2.5. Let c : $G \rightarrow \mathbf{R}$ be a continuous cocycle, where $G$ is a locally compact groupoid which has a Haar system. Assume that the groupoid $G$ is proper. Then, there exists a continuous function $b: G^{(0)} \rightarrow \mathbf{R}$ such that $c(\gamma)=b \circ r(\gamma)-b \circ s(\gamma)$.

Proof. Let $\pi: G^{(0)} \rightarrow G \backslash G^{(0)}$ be the quotient map. Since $G \backslash G^{(0)}$ is paracompact and $\pi$ is an open map, we can apply [3, Appendice 1, Lemme 1]: there exists $F: G^{(0)} \rightarrow \mathbf{R}^{+}$continuous such that:

a) $F$ is not identically zero on any equivalence class modulo $G$;

b) for every compact set $K \subset G \backslash G^{(0)}$, the intersection of $\pi^{-1}(K)$ with the support of $F$ is compact.

Let $\lambda=\left(\lambda^{x}\right)$ be a Haar system for $G$. The function $h: G^{(0)} \rightarrow \mathbf{R}^{+}$defined by $h(x)=$ $\int(F \circ s)(\gamma) d \lambda^{x}(\gamma)$ for $x \in G^{(0)}$ is well defined and strictly positive. Moreover, it is constant on the equivalence classes. If we replace $F$ by $F / h$, we obtain a function (still denoted by $F$ ) which satisfies a), b) and the following condition:

c) $\int(F \circ s)(\gamma) d \lambda^{x}(\gamma)=1$ for all $x \in G^{(0)}$.

Such a function is also constructed in [29, Section 6] where it is called a cutoff function. Then, the integral

$$
b(x)=\int(F \circ s)(\gamma) c(\gamma) d \lambda^{x}(\gamma)
$$

is well defined and defines a continuous function such that $c(\gamma)=b \circ r(\gamma)-b \circ s(\gamma)$.

The technique of the second result is also adapted from [3]. It can also be found in [1, Appendix A.1] in the case of a proper Borel equivalence relation.

Lemma 2.6. Let $G$ be a proper locally compact groupoid with Haar system $\lambda$. Then,

(i) the quotient map $\pi: G^{(0)} \rightarrow G \backslash G^{(0)}$ carries a continuous $\pi$-system of measures $\dot{\lambda}$ defined by

$$
\dot{\lambda}(f)(\dot{x})=\int(f \circ s) d \lambda^{x}
$$

where $f \in C_{c}\left(G^{(0)}\right), x \in G^{(0)}$ and $\dot{x}=\pi(x)$;

(ii) a Radon measure $\mu$ on $G^{(0)}$ is of the form $m \circ \dot{\lambda}$ for some Radon measure $m$ on $G \backslash G^{(0)}$ if and only if the measure $\mu \circ \lambda$ on $G$ is symmetric, i.e. satisfies $\mu \circ \lambda=(\mu \circ \lambda)^{-1}$.

Proof. Since this is classical, we just give a sketch of the proof. The integral in (i) is well defined because $G$ is proper. The necessity of the symmetry of $m \circ \dot{\lambda} \circ \lambda$ is given by Fubini's theorem. The construction of $m$ uses the cutoff function $F$ of the previous lemma. Explicitly, for $f \in C_{c}\left(G \backslash G^{(0)}\right), m(f)=\mu(F(f \circ \pi))$. 
One can combine this lemma and Lemma 3.2 given below to describe all quasi-invariant measures of a proper groupoid: up to equivalence, they are given by measures on the quotient space.

We can now construct the composition of two correspondences. Let $\left(G_{i}, \lambda_{i}\right)$, where $i=1,2,3$, be locally compact groupoids with Haar systems, $(X, \alpha)$ a correspondence from $G_{1}$ to $G_{2}$ with module $\Delta_{X}: G_{1} \ltimes X / G_{2} \rightarrow \mathbf{R}_{+}^{*}$ and $(Y, \beta)$ a correspondence from $G_{2}$ to $G_{3}$ with module $\Delta_{Y}: G_{2} \ltimes Y / G_{3} \rightarrow \mathbf{R}_{+}^{*}$. One constructs a correspondence $(Z, \tau)$ from $G_{1}$ to $G_{3}$ with module $\Delta: G_{1} \ltimes Z / G_{3} \rightarrow \mathbf{R}_{+}^{*}$ as follows. As a $\left(G_{1}, G_{3}\right)$-space, $Z$ is the usual composition $(X * Y) / G_{2}$, where $X * Y$ is the fibered product over $G_{2}^{(0)}$ and $G_{2}$ acts by $(x, y) h=\left(x h, h^{-1} y\right)$. One defines the system of measures $\left(\beta_{u} \circ \alpha\right)_{u \in G_{3}^{(0)}}$ for the map $s: X * Y \rightarrow G_{3}^{(0)}$ sending $(x, y)$ to $s_{Y}(y)$ by

$$
\int f d\left(\beta_{u} \circ \alpha\right)=\int f(x, y) d \alpha_{r(y)}(x) d \beta_{u}(y)
$$

for $f \in C_{c}(X * Y)$. Let $\pi: X * Y \rightarrow Z=X * Y / G_{2}$ denote the quotient map. It carries the system of measures $\lambda=\left(\lambda_{z}\right)_{z \in Z}$ defined by

$$
\int f d \lambda_{\pi(x, y)}=\int f\left(x h, h^{-1} y\right) d \lambda_{2}^{s(x)}(h) .
$$

Since $G_{2}$ acts properly on $X$, the groupoid $(X * Y) \rtimes G_{2}$ is proper. We view $\Delta_{Y}$ as a cocycle on $(X * Y) \rtimes G_{2}$ and use Lemma 2.5 to trivialize it: there exists a continuous $b: X * Y \rightarrow \mathbf{R}_{+}^{*}$ such that for all $(x, y) \in X * Y$ and $h \in G_{2}$ such that $s(h)=r(y)$, the equality

$$
\Delta_{Y}(h, y)=b\left(x h^{-1}, h y\right) / b(x, y)
$$

holds. Moreover, since $\Delta_{Y}(h, y)$ depends only on the class $\dot{y}$ in $Y / G_{3}$, we may assume that $b(x, y)$ depends only on $(x, \dot{y})$. Let us fix $u \in G_{3}^{(0)}$. The measure $b\left(\beta_{u} \circ \alpha\right)$ on $X * Y$ satisfies the condition (ii) of Lemma 2.6 with respect to $\left((X * Y) \rtimes G_{2}, \lambda_{2}\right)$. Hence there exists a measure $\tau_{u}$ on $Z=X * Y / G_{2}$ such that

$$
b\left(\beta_{u} \circ \alpha\right)=\tau_{u} \circ \lambda .
$$

Note that $\tau_{u}$ is supported on $Z_{u}=\pi\left(X * s_{Y}^{-1}(u)\right)$. This defines the desired system $\tau=\left(\tau_{u}\right)_{u \in G_{3}^{(0)}}$. Since the measures $b\left(\beta_{u} \circ \alpha\right)$ are $\Delta$-measures with respect to $\left(G_{1}, \lambda_{1}\right)$, where

$$
\Delta(\gamma,(x, y))=b(\gamma x, y)^{-1} \Delta_{X}(\gamma, x) b(x, y),
$$

one deduces (see Proposition 3.1 below) that $\Delta(\gamma,(x, y))$ depends only on $(\gamma, \pi(x, y))$ and that the measures $\tau_{u}$ are $\Delta$-measures with respect to $\left(G_{1}, \lambda_{1}\right)$. We write $Z=X *_{G_{2}} Y$ and $\tau=\alpha *_{G_{2}} \beta$.

We state without a proof the expected result:

Theorem 2.7 ([10]). The construction of the $C^{*}$-algebra of a locally compact groupoid with Haar system is functorial. More precisely, given $\left(G_{i}, \lambda_{i}\right)$, where $i=1,2,3,(X, \alpha)$ and $(Y, \beta)$ as above, the $C^{*}$-correspondence $C^{*}\left(X *_{G_{2}} Y, \alpha *_{G_{2}} \beta\right)$ is isomorphic to the composition of the $C^{*}$-correspondences $C^{*}(X, \alpha) \otimes_{C^{*}\left(G_{2}, \lambda_{2}\right)} C^{*}(Y, \beta)$.

\section{Induced representations}

As we have seen, a correspondence $(X, \alpha)$ from $(H, \beta)$ to $(G, \lambda)$ gives a $C^{*}$-correspondence $C^{*}(X, \alpha)$ from $C^{*}(H, \beta)$ to $C^{*}(G, \lambda)$, hence it induces a map from $\operatorname{Rep}(G)$ to $\operatorname{Rep}(H)$, where $\operatorname{Rep}(G)$ is the set of equivalence classes of representations of $G$. The purpose of this section is to describe this map without passing through the $C^{*}$-algebras. Recall from [24] that a representation of $G$ is a pair $(m, \mathcal{H})$, where $m$ is a transverse measure class on $G$ and $\mathcal{H}$ is a measurable $G$ Hilbert bundle. Since we have fixed a Haar system $\lambda$ for $G$, the transverse measure class $m$ is given by a quasi-invariant measure $\mu$ on $G^{(0)}$. We denote by $\Delta_{\mu}: G \rightarrow \mathbf{R}_{+}^{*}$ its module. 


\subsection{Disintegration of quasi-invariant measures}

The following proposition is a slight variation of [1, Corollary 5.3.11]. It generalizes [21, Subsection I.3.21] and plays a crucial role in the construction of induced representations. We use the notation $\lambda_{1}, \lambda_{2}$ introduced before Definition 2.2.

Proposition 3.1. Let $(G, \lambda)$ be a locally compact groupoid with Haar system. Let $X, Y$ be left $G$-spaces and $\pi: X \rightarrow Y$ be a continuous $G$-equivariant surjection. Let $\nu$ be a $\sigma$-finite measure on $X$ and let $\nu=\int \rho_{y} d \mu(y)$ a disintegration of $\nu$ along $\pi$.

(i) If $\nu$ is quasi-invariant with respect to $(G, \lambda)$, then $\mu$ is quasi-invariant with respect to $(G, \lambda)$ and for $\left(\mu \circ \lambda_{1}^{Y}\right)$-a.e. $(\gamma, y) \in G * Y, \gamma \rho_{y} \sim \rho_{\gamma y}$. More precisely, let $\Delta_{X}: G \ltimes X \rightarrow \mathbf{R}_{+}^{*}$ be such that

$$
\nu \circ \lambda_{1}^{X}=\Delta_{X}\left(\nu \circ \lambda_{2}^{X}\right) .
$$

Then there exist measurable functions $\Delta_{Y}: G \ltimes Y \rightarrow \mathbf{R}_{+}^{*}$ and $\delta: G \ltimes X \rightarrow \mathbf{R}_{+}^{*}$ such that

$$
\begin{aligned}
& \mu \circ \lambda_{1}^{Y}=\Delta_{Y}\left(\mu \circ \lambda_{2}^{Y}\right), \\
& \gamma \rho_{y}=\delta\left(\gamma^{-1}, \cdot\right) \rho_{\gamma y}
\end{aligned}
$$

and they are related by

$$
\Delta_{X}(\gamma, x)=\delta(\gamma, x) \Delta_{Y}(\gamma, \pi(x))
$$

for $\nu \circ \lambda_{1}^{X}$ a.e. $(\gamma, x) \in G * X$.

(ii) Conversely, suppose that there exist measurable functions $\Delta_{Y}: G \ltimes Y \rightarrow \mathbf{R}_{+}^{*}$ and $\delta$ : $G \ltimes X \rightarrow \mathbf{R}_{+}^{*}$ such that (3.2) and (3.3) hold. Then $\nu=\mu \circ \rho$ is a $\Delta_{X}$-measure, where $\Delta_{X}$ is defined by (3.4).

Proof. We start with an observation. Let us introduce the map

id $\times \pi: G \ltimes X \rightarrow G \ltimes Y$.

We endow it with the system of measures $1 \otimes \rho$. By construction, we have the commutation relation

$$
\lambda_{2}^{Y} \circ(1 \otimes \rho)=\rho \circ \lambda_{2}^{X} .
$$

However, a similar commutation relation for $\lambda_{1}^{X}$ and $\lambda_{1}^{Y}$ requires the relation (3.3). More precisely, if (3.3) holds, a lengthy but straightforward computation gives

$$
\lambda_{1}^{Y} \circ(1 \otimes \rho)=\delta^{-1}\left(\rho \circ \lambda_{1}^{X}\right) .
$$

Let us first prove the assertion (ii). We assume that (3.2) and (3.3) hold. Starting from the equality

$$
\nu \circ \lambda_{1}^{X}=\mu \circ \rho \circ \lambda_{1}^{X},
$$

and identifying functions and the corresponding multiplication operators, we have

$$
\begin{aligned}
\nu \circ \lambda_{1}^{X} \circ \delta^{-1} & =\mu \circ \rho \circ \lambda_{1}^{X} \circ \delta^{-1}=\mu \circ \lambda_{1}^{Y} \circ(1 \otimes \rho)=\mu \circ \lambda_{2}^{Y} \circ \Delta_{Y} \circ(1 \otimes \rho) \\
& =\mu \circ \lambda_{2}^{Y} \circ(1 \otimes \rho) \circ\left(\Delta_{Y} \circ(\mathrm{id} \times \pi)\right)=\mu \circ \rho \circ \lambda_{2}^{X} \circ\left(\Delta_{Y} \circ(\mathrm{id} \times \pi)\right) \\
& =\nu \circ \lambda_{2}^{X} \circ\left(\Delta_{Y} \circ(\mathrm{id} \times \pi)\right) .
\end{aligned}
$$


Thus, we can write $\nu \circ \lambda_{1}^{X}=\Delta_{X}\left(\nu \circ \lambda_{2}^{X}\right)$ where

$$
\Delta_{X} \delta^{-1}=\Delta_{Y} \circ(\mathrm{id} \times \pi) \quad \text { or equivalently } \quad \Delta_{X}(\gamma, x)=\Delta_{Y}(\gamma, \pi(x)) \delta(\gamma, x) .
$$

This shows that $\nu$ is a $\Delta_{X}$-measure with respect to $(G, \lambda)$.

Let us prove the assertion (i). We assume that (3.1) holds. As pseudo-images of the equivalent measures $\mu \circ \lambda_{2}^{X}$ and $\mu \circ \lambda_{1}^{X}$ under $i d \times \pi: G \ltimes X \rightarrow G \ltimes Y$, the measures $\mu \circ \lambda_{2}^{Y}$ and $\mu \circ \lambda_{1}^{Y}$ are equivalent. Therefore there exists a measurable function $\Delta_{Y}: G \ltimes Y \rightarrow \mathbf{R}_{+}^{*}$ such that (3.2) holds. We are going to compare two disintegrations of the measure $\nu \circ \lambda_{1}^{X}$ on $G \ltimes X$ along $i d \times \pi: G \ltimes X \rightarrow G \ltimes Y$, taking the same measure $\mu \circ \lambda_{1}^{Y}$ as base measure. The first one is obtained by applying the inverse map of $G \ltimes X$ to the disintegration

$$
\nu \circ \lambda_{2}^{X}=\left(\mu \circ \lambda_{2}^{Y}\right) \circ(1 \otimes \rho) .
$$

This yields after some computation:

$$
\nu \circ \lambda_{1}^{X}=\left(\mu \circ \lambda_{1}^{Y}\right) \circ \Phi \quad \text { where } \quad \Phi(f)(\gamma, y)=\int f\left(\gamma, \gamma^{-1} x\right) d \rho_{\gamma y}(x) .
$$

The second disintegration is obtained from the relations (3.1) and (3.2):

$$
\begin{aligned}
\nu \circ \lambda_{1}^{X} & =\nu \circ \lambda_{2}^{X} \circ \Delta_{X}=\mu \circ \rho \circ \lambda_{2}^{X} \circ \Delta_{X}=\mu \circ \lambda_{2}^{Y} \circ(1 \otimes \rho) \circ \Delta_{X} \\
& =\mu \circ \lambda_{1}^{Y} \circ \Delta_{Y}^{-1} \circ(1 \otimes \rho) \circ \Delta_{X} .
\end{aligned}
$$

By uniqueness of the disintegration along the map $i d \times \pi$, we get

$$
\Phi=\Delta_{Y}^{-1} \circ(1 \otimes \rho) \circ \Delta_{X}
$$

or equivalently, for all $f \in C_{c}(G \ltimes X)$ and $\nu \circ \lambda_{1}^{Y}$ a.e. $(\gamma, y) \in G \ltimes Y$,

$$
\int f\left(\gamma, \gamma^{-1} x\right) d \rho_{\gamma y}(x)=\int f(\gamma, x) \frac{\Delta_{X}(\gamma, x)}{\Delta_{Y}(\gamma, \pi(x))} d \rho_{y}(x)
$$

This gives the formula

$$
\gamma^{-1} \rho_{\gamma y}=\delta(\gamma, \cdot) \rho_{y}, \quad \text { where } \quad \delta(\gamma, x)=\frac{\Delta_{X}(\gamma, x)}{\Delta_{Y}(\gamma, \pi(x))} .
$$

\subsection{Construction of the induced quasi-invariant measure}

Let us first state and prove a result which is the measurable version of Lemma 2.5.

Lemma 3.2. Let $c: G \rightarrow \mathbf{R}$ be a measurable cocycle, where $G$ is a locally compact groupoid endowed with a Haar system and a quasi-invariant measure. Assume that the groupoid $G$ is proper. Then, there exists a measurable function $b: G^{(0)} \rightarrow \mathbf{R}$ such that $c(\gamma)=b \circ r(\gamma)-b \circ s(\gamma)$ for all $\gamma \in G$.

Proof. We denote by $R$ be the graph of the equivalence relation on $G^{(0)}$ defined by $G$ and by $G^{\prime}$ the isotropy bundle of $G$, i.e. the subgroupoid defined by $r(\gamma)=s(\gamma)$ (it is the union of the isotropy groups). Since $G$ is proper, the isotropy groups $G(x)$, where $x \in G^{(0)}$, are compact. Therefore, the restriction of $c$ to $G(x)$ and to $G^{\prime}$ is trivial. Since $R$ can be identified with the quotient $G^{\prime} \backslash G$, there exists a measurable cocycle $\underline{c}: R \rightarrow \mathbf{R}$ such that for all $\gamma \in G$, $c(\gamma)=\underline{c}(r(\gamma), s(\gamma))$. Since $R$ is a closed subset of $G^{(0)}$, the quotient map $\pi: G^{(0)} \rightarrow G \backslash G^{(0)}$ has a Borel section $\sigma$ (see [20, Theorem 2.1]). The function $b: G^{(0)} \rightarrow \mathbf{R}$ defined by $b(x)=\underline{c}(x, \sigma(x))$ is a measurable coboundary for $c$. 
We now begin the construction of the representation $(\mu, \underline{\mathcal{H}})$ of $(H, \beta)$ induced by the representation $(\mu, \mathcal{H})$ of $(G, \lambda)$ through the correspondence $(X, \bar{\alpha})$. We first define the quasi-invariant measure $\mu$. The module of $\mu$ is denoted by $\Delta_{\mu}$. Using [19, Theorem 3.2], we choose $\Delta_{\mu}$ so that it is a strict homomorphism. According to Proposition 3.1 applied to the $G$-map $s_{X}: X \rightarrow G^{(0)}$, the measure $\mu \circ \alpha$ on $X$ is a $\Delta_{X}$-measure on $X$ with respect to $(G, \lambda)$, where $\Delta_{X}(x, \gamma)=\Delta_{\mu}(\gamma)$. We use the same idea as in Section 2.5, namely we trivialize the cocycle $\Delta_{X}$ on the proper groupoid $X \rtimes G$. We apply Lemma 3.2 to obtain a measurable function $b: X \rightarrow \mathbf{R}_{+}^{*}$ such that $\Delta_{X}(x, \gamma)=b(x \gamma) / b(x)$. Then, the measure $b(\mu \circ \alpha)$ is an invariant measure with respect to $(X \rtimes G, \lambda)$. According to Lemma 2.6, there is a measure $m$ on $X / G$ such that

$$
b(\mu \circ \alpha)=m \circ \dot{\lambda},
$$

where we have used the same notation as in the lemma. Here,

$$
\dot{\lambda}(f)(\dot{x})=\int f(x \gamma) d \lambda^{s(x)}(\gamma) \quad \text { for } \quad f \in C_{c}(X) .
$$

Next, we study the invariance property of $m$ with respect to $(H, \beta)$. Since the $\alpha_{u}$ 's are $\Delta$ measures, so is $\mu \circ \alpha$. This implies that the measure $m \circ \dot{\lambda}$ satisfies:

$$
m \circ \dot{\lambda} \circ \beta_{1}=\frac{b \circ r}{b \circ s} \Delta\left(m \circ \dot{\lambda} \circ \beta_{2}\right) \text {. }
$$

Since the system $\dot{\lambda}$ is invariant under $H$, Proposition 3.1 gives that

$$
m \circ \beta_{1}=\Delta_{m}\left(m \circ \beta_{2}\right),
$$

where

$$
\Delta_{m}(h, \dot{x})=\frac{b(h x)}{b(x)} \Delta(h, \dot{x})
$$

The last step is to pass from the measure $m$ on $X / G$ to the measure $\mu$ on $H^{(0)}$. We just choose a pseudo-image $\underline{\mu}$ of $m$ and disintegrate $m$ along $\dot{r}: X / G \rightarrow H^{(0)}$ :

$$
m=\int \rho^{u} d \underline{\mu}(u)
$$

According to Proposition 3.1, $\underline{\mu}$ is quasi-invariant and we have cocycles $\Delta_{\mu}: H \rightarrow \mathbf{R}_{+}^{*}$ and $\delta: H \ltimes X / G \rightarrow \mathbf{R}_{+}^{*}$ such that $\underline{\mu}$ is quasi-invariant with module $\Delta_{\underline{\mu}}$ and

$$
\begin{aligned}
& h \rho^{s(h)}=\delta(h, \cdot) \rho^{r(h)}, \\
& \Delta_{\underline{\mu}}(h)=\Delta_{m}(h, \dot{x}) \delta(h, \dot{x}) .
\end{aligned}
$$

\subsection{Construction of the induced Hilbert bundle}

Given a representation $(\mu, \mathcal{H})$ of $(G, \lambda)$, we have constructed in the previous subsection the induced quasi-invariant measure $\mu$ of $(H, \beta)$. Let us now construct the induced $H$-Hilbert bundle $\underline{\mathcal{H}}$. For $x \in X$ we denote by $G(x)=\{\gamma \in G: \gamma x=x\}$ the stabilizer of $x$ and by $\kappa_{x}$ its normalized Haar measure. Then $\kappa=\left(\kappa_{x}\right)_{x \in X}$ is a Borel Haar system for the the isotropy group bundle $G(X)$ of $X \rtimes G$ (see for example [25, Lemma 1.5]). We view the measurable $G$-Hilbert bundle $X * \mathcal{H}$ over $X$ as a $X \rtimes G$-Hilbert bundle. 
Definition 3.3. Let $\mathcal{H}$ be a measurable $G$-Hilbert bundle with bundle map $p: \mathcal{H} \rightarrow G^{(0)}$. We define its fixed-point bundle as

$$
\mathcal{H}^{G}=\{\xi \in \mathcal{H}: \gamma p(\xi)=p(\xi) \Rightarrow L(\gamma) \xi=\xi\} .
$$

We shall use the following easy result.

Proposition 3.4. Let $\mathcal{H}$ be a measurable G-Hilbert bundle.

- $\mathcal{H}^{G}$ is a measurable $G$-Hilbert subbundle of $\mathcal{H}$.

- If $G$ is a proper groupoid, integration over the isotropy subgroups defines a bundle projection of $\mathcal{H}$ onto $\mathcal{H}^{G}$.

We define integration over the isotropy subgroup $G(x)$, where $x \in G^{(0)}$, as the orthogonal projection of $\mathcal{H}_{x}$ onto $\mathcal{H}_{x}^{G(x)}$

$$
P_{x} \xi=\int L(\gamma) \xi d \kappa_{x}(\gamma) \quad \text { if } \quad \xi \in \mathcal{H}_{x}
$$

where $\kappa_{x}$ is the normalized Haar measure of $G(x)$. The family $P=\left(P_{x}\right)_{x \in G^{(0)}}$ defines the bundle projection.

Going back to our previous situation, we consider the fixed-point bundle $\mathcal{H}^{\prime}=(X * \mathcal{H})^{X \rtimes G}$ of $X * \mathcal{H}$. The fiber above $x \in X$ is:

$$
\mathcal{H}_{x}^{\prime}=\left\{\xi \in \mathcal{H}_{s(x)}: \forall h \in G(x), L(h) \xi=\xi\right\} .
$$

This bundle is stable under the action of $G$ and is a proper $G$-space. We denote by $\mathcal{K}=\mathcal{H}^{\prime} / G$ the quotient space.

Proposition 3.5. The quotient space $\mathcal{K}=\mathcal{H}^{\prime} / G$ is a measurable Hilbert bundle over $X / G$, where the bundle map $p: \mathcal{K} \rightarrow X / G$ sends the class $[x, \xi]$ of $(x, \xi)$ to the class $\dot{x}$ of $x$.

Proof. A choice of $x \in X$ defines a bijection $\varphi_{x}: \mathcal{H}_{x}^{\prime} \rightarrow \mathcal{K}_{\dot{x}}$ such that $\varphi_{x}(\xi)=[x, \xi]$. We use this bijection to carry to $\mathcal{K}_{\dot{x}}$ the Hilbert space structure of $\mathcal{H}_{x}^{\prime}$. This Hilbert space structure does not depend on the choice of $x$ in its class: suppose that $y=x \gamma$. The isometry $L(\gamma): \mathcal{H}_{s(\gamma)} \rightarrow \mathcal{H}_{r(\gamma)}$ sends $\mathcal{H}_{y}^{\prime}$ onto $\mathcal{H}_{x}^{\prime}$ and we have $\varphi_{y}=\varphi_{x} \circ L(\gamma)$. Let $P_{x}: \mathcal{H}_{s(x)} \rightarrow \mathcal{H}_{x}^{\prime}$ be the orthogonal projection. It satisfies $L(\gamma) \circ P_{y}=P_{x} \circ L(\gamma)$. As it is a subbundle of $X * \mathcal{H}, \mathcal{H}^{\prime}$ has a measurable Hilbert bundle structure and so has the quotient bundle $\mathcal{K}$ : we shall say that a section $\xi$ of $p: \mathcal{K} \rightarrow X / G$ is measurable if there is a measurable section $\sigma$ for the quotient map $X \rightarrow X / G$ and a measurable section $\eta$ of the bundle $\mathcal{H}^{\prime}$ such that $\xi(\dot{x})=[\eta \circ \sigma(\dot{x})]$.

The Hilbert bundle $X * \mathcal{H}$ is endowed with the unitary $H$-action: $h(x, \xi)=(h x, \xi)$. Since this $H$-action commutes with the action of $G$, the subbundle $\mathcal{H}^{\prime}$ is invariant under $H$ and the quotient $\mathcal{K}$ is also a $H$-Hilbert bundle. The last step is to go from the $H$-Hilbert bundle $\mathcal{K}$ over $X / G$ to a $H$-Hilbert bundle $\underline{\mathcal{H}}$ over $H^{(0)}$. We use the disintegration $\nu=\int \rho^{u} d \underline{\mu}(u)$ of the measure $\nu$ given earlier. We define for $u \in H^{(0)}$ :

$$
\underline{\mathcal{H}}_{u}=L^{2}\left(X / G, \rho^{u}, \mathcal{K}\right) .
$$

As explained earlier, the measures $\nu$ on $X / G$ and $\mu$ on $H^{(0)}$ are quasi-invariant and there exists a measurable function $\delta: H \ltimes X / G \rightarrow \mathbf{R}_{+}^{*}$ such that $h \rho^{s(h)}=\delta\left(h^{-1}, \cdot\right) \rho^{r(h)}$. The measurable structure of the bundle $\left(\underline{\mathcal{H}}_{u}\right)_{u \in H^{(0)}}$ is provided by the family of measurable sections $u \mapsto \xi_{u}$, where $\xi$ is a measurable section of the bundle $\mathcal{K} \rightarrow X / G$ and $\xi_{u}(\dot{x})=\xi(\dot{x})$ if $r(\dot{x})=u$. For $h \in H$, we define $L(h): \underline{\mathcal{H}}_{s(h)} \rightarrow \underline{\mathcal{H}}_{r(h)}$ by

$$
\left(L(h) \xi_{s(h)}\right)(\dot{x})=\delta^{1 / 2}\left(h^{-1}, \dot{x}\right) \xi_{s(h)}\left(h^{-1} \dot{x}\right) .
$$

These are unitary operators and $\underline{\mathcal{H}}$ is the desired $H$-Hilbert bundle. 


\subsection{Examples}

Let us spell out the above construction in the classical case 2.3.c, where $H$ is a closed subgroup of $G$ and $X=G$ is viewed as a correspondence from $G$ to $H$ (note that we considered earlier a correspondence from $H$ to $G$ ). To construct the measure $m$ on $G / H$, we find $b: G \rightarrow \mathbf{R}_{+}^{*}$ such that $b(x h)=b(x) \delta_{H}(h)$ for $(x, h) \in G \times H$. According to Lemma 2.6(ii), we can write

$b \lambda^{-1}=m \circ \dot{\beta}$ where $\dot{\beta}(f)(\dot{x})=\int f(x h) d \beta(h)$. Then $m$ is quasi-invariant with respect to $(G, \lambda)$.

Since $H^{(0)}$ is reduced to one element $e$, we have $m=\rho^{e}$ in equation (3.6). The equations (3.5) and (3.7) become respectively $\Delta_{m}(\gamma, \dot{x})=b(\gamma x) / b(x)$ and $\gamma m=\delta(\gamma, \cdot) m$ where according to equation (3.8), $\delta: G \ltimes(G / H) \rightarrow \mathbf{R}_{+}^{*}$ satisfies $\delta_{G}(\gamma)=\frac{b(\gamma x)}{b(x)} \delta(\gamma, \dot{x})$. The $G$-Hilbert space induced by a $H$-Hilbert space $\mathcal{H}$ is $L^{2}(G / H, m,(G \times \mathcal{H}) / H)$.

Let $H_{1}, H_{2}$ be closed subgroups of a locally compact group $G$. We denote by $\beta_{1}, \beta_{2}$ and $\delta_{1}, \delta_{2}$ the Haar measures and modules respectively. Then $(X, \alpha)=\left(G, \lambda^{-1}\right)$ can also be viewed as a correspondence from $\left(H_{1}, \beta_{1}\right)$ to $\left(H_{2}, \beta_{2}\right)$. As we have seen earlier, $\alpha$ is a $\Delta$-measure with respect to $\left(H_{1}, \beta_{1}\right)$, where $\Delta(h)=\delta_{1}(h) / \delta_{G}(h)$ for $h \in H_{1}$. Given a $H_{2}$-Hilbert space $\mathcal{H}$, the induced Hilbert space is just as above $\underline{\mathcal{H}}=L^{2}\left(G / H_{2}, m,(G \times \mathcal{H}) / H_{2}\right)$ and the action of $H_{1}$ is given by the same formula as before. If we assume that $H_{1}$ acts properly on $G / H_{2}$, this representation can be decomposed over $H_{1} \backslash G / H_{2}$. This is best understood at the level of the correspondence $X$. Let $\pi: X \rightarrow H_{1} \backslash G / H_{2}$ be the quotient map. For a double class $d=H_{1} x H_{2}$, we also write $X_{d}=$ $\pi^{-1}(d)=H_{1} x H_{2}$. We view $X_{d}$ as a correspondence from $H_{1}$ to $H_{2}$. It is equipped with a measure $\alpha_{d}$ coming from a disintegration of $\alpha$ along $\pi$. One observes that $X_{d} / H_{2}$ and $H_{1} /\left(H_{1} \cap x H_{2} x^{-1}\right)$ are isomorphic $H_{1}$-spaces (and their measures match). Therefore, the $H_{1}$-Hilbert space $\underline{\mathcal{H}}_{d}$ induced through $X_{d}$ from $\mathcal{H}$ is easily identified: it is isomorphic to the $H_{1}$-Hilbert space induced from the representation $\mathcal{H}^{x}$ of its subgroup $H_{1} \cap x H_{2} x^{-1}$, given by $\mathcal{H}^{x}=\mathcal{H}$ and $L^{x}(h)=L\left(x^{-1} h x\right)$ for $h \in H_{1} \cap x H_{2} x^{-1}$. Then, the $H_{1}$-Hilbert space $\underline{\mathcal{H}}$ is a direct integral of the $\underline{\mathcal{H}}_{d}$ 's over $H_{1} \backslash G / H_{2}$. This is the content of G. Mackey's subgroup theorem [16, Theorem 12.1].

\section{Hypergroupoids}

G. Mackey introduced virtual subgroups (and virtual groups) to the purpose of generalizing the theory of induced representations. From the $C^{*}$-algebraic point of view, induction only requires a $C^{*}$-correspondence. Groupoid correspondences and equivalences suggest to consider more general objects, namely hypergroupoids, and their $C^{*}$-algebras, to construct induced representations. Let us see how hypergroupoid $C^{*}$-algebras enter into our framework. When $X$ is a $(G, H)$-groupoid equivalence, $X$ as a left free and proper $G$-space determines $H$ up to isomorphism. Indeed $H$ is isomorphic to the groupoid $(X * X) / G$. Moreover, a continuous $G$ equivariant system of measures $\alpha$ on $X$ defines a Haar system on $(X * X) / G$. Let us assume that $X$ is a proper, but not necessarily free, $G$-space endowed with a continuous $G$-equivariant system of measures $\alpha$. It is shown in [11] that, although $H=(X * X) / G$ is no longer a groupoid, the *-algebra $C_{c}(H)$ can be defined by the same formulas as in the case of a free and proper $G$ space. The representations of $G$ extend to representations of $C_{c}(H)$ and there is a least $C^{*}$-norm making all these representations continuous. We denote by $C_{G}^{*}(H)$ the corresponding completion of $C_{c}(H)$. One can also complete $C_{c}(X)$ into a $C^{*}$-correspondence $C^{*}(X)$ from $C^{*}(G)$ to $C_{G}^{*}(H)$. Thus one can induce representations from $C_{G}^{*}(H)$ to $C^{*}(G)$. These are well known constructions in the theory of unitary representations of groups. The usual situation is a pair $(G, K)$ where $K$ is a compact subgroup of a locally compact group $G$. Then $X=G / K$ is a proper left $G$-space which has an invariant measure. The corresponding hypergroupoid $H=(X * X) / G$ is isomorphic to the double coset hypergroup $K \backslash G / K$. As we have seen, one can induce representations 
of $G$ to representations of the *-algebra $C_{c}(H)$. The converse problem is more interesting: which representations of $C_{c}(H)$ induce representations of $G$ ? These problems are studied in the framework of induced representations of hypergroups in [6] and in $[7,8]$, where the author also uses Rieffel's $C^{*}$-algebraic machinery.

The structure of $H=(X * X) / G$, where $G$ is a locally compact groupoid and $X$ is a (say left) proper $G$-space endowed with a continuous $G$-equivariant $r_{X}$-system $\alpha$ is that of a locally compact hypergroupoid with Haar system, which we are going to define in this section. Hypergroupoids of that form will be termed spatial hypergroupoids. Hypergroups are hypergroupoids whose unit space is reduced to one point. Double coset spaces $K \backslash G / K$ as above are spatial hypergroups. The main difference between the hypergroups considered here and the classical theory of locally compact hypergroups of Ch. Dunkl [5], R. Jewett [12] and R. Spector [28] is that we assume the existence of a Haar system, which allows the definition of their $C^{*}$-algebras just as in the case of groups, while the classical theory deals with measure algebras. The definition of a locally compact hypergroupoid with Haar system which will be proposed in this section is a tentative one. It is inspired by the definition of a hypergroup given by $[5,12,28]$ and is rather close in spirit to [13]. Its main virtue is to cover the spatial hypergroupoids as above and the locally compact hypergroups which have a Haar measure; moreover, it makes the theory a development of the case of locally compact groupoids with Haar systems.

The idea of the definition is very simple: we take the usual definition of a locally compact groupoid $H$ but where the product of two composable elements $x, y$ is no longer a third element but a probability measure $x * y$ with compact support. Given a locally compact space $X$, $P(X)$ denotes its space of probability measures and given $x \in X, \delta_{x}$ denotes the point mass at $x$. For the clarity of the exposition, we present firstly the axioms concerning the product and the involution and secondly the axioms concerning the Haar system. However, we are only concerned with locally compact hypergroupoids with Haar systems, that is, hypergroupoids which satisfy the two sets of axioms.

Definition 4.1. A locally compact hypergroupoid is defined as a pair $\left(H, H^{(0)}\right)$ of locally compact spaces, range and source maps $r, s: H \rightarrow H^{(0)}$ assumed to be continuous, open and surjective, a continuous injection $i: H^{(0)} \rightarrow H$ such that $r \circ i$ and $s \circ i$ are the identity map, a continuous involution inv : $h \mapsto h^{*}$ of $H$ such that $r \circ$ inv $=s$ and a product map $m: H^{(2)} \rightarrow P(H)$, where $H^{(2)}$ is the set of composable pairs, i.e. pairs $(x, y) \in H \times H$ with $s(x)=r(y)$ (one defines similarly $H^{(3)}$ ) such that:

(i) the support of $m(x, y)$ is a compact subset of $H_{s(y)}^{r(x)}$;

(ii) for all $(x, y, z) \in H^{(3)}$, we have $\int m(x, \cdot) d m(y, z)=\int m(\cdot, z) d m(x, y)$;

(iii) for all $x \in H, m(r(x), x)=m(x, s(x))=\delta_{x}$;

(iv) for all $(x, y) \in H^{(2)}, m(x, y)^{*}=m\left(y^{*}, x^{*}\right)$, where $m(x, y)^{*}$ is the image of the measure $m(x, y)$ by the involution;

(v) $x=y^{*}$ if and only if the support of $m(x, y)$ meets $i\left(H^{(0)}\right)$;

(vi) for all $f \in C_{c}(H)$ and $\epsilon>0$, there exists a neighborhood $U$ of $i\left(H^{(0)}\right)$ in $H$ such that $\left|f(x)-f\left(y^{*}\right)\right| \leq \epsilon$ as soon as the support of $m(x, y)$ meets $U$;

(vii) for all $x \in H$, the left translation operator $L(x)$ defined by

$$
(L(x) f)(y)=f\left(x^{*} * y\right):=\int f d m\left(x^{*}, y\right)
$$

sends $C_{c}\left(H^{s(x)}\right)$ into $C_{c}\left(H^{r(x)}\right)$. 
Remark 4.2. When the measures $m(x, y)$ are point masses, one retrieves the product and the inverse map of a groupoid. Note however that, as explained below, the continuity of the product is not assumed. We suspect that adding the second set of axioms concerning Haar systems will force the continuity of the product but we have not checked this. Our axioms are modelled after the definition of a locally compact hypergroup, as given in [12] (where it is called a convo) or [2, Subsection 1.1.2]. They are tailored to fit our main class of examples, namely spatial hypergroupoids, described below in Theorem 4.5. Simple examples of spatial hypergroupoids, for example the hypergroupoid constructed from the action of $\mathbf{Z} / 2 \mathbf{Z}$ on $\mathbf{R}$ by the map $x \mapsto-x$, show that the product map is not necessarily continuous, in the sense that for $f \in C_{c}(H)$, the map $(x, y) \mapsto f(x * y):=\int f d m(x, y)$ may fail to be continuous on $H^{(2)}$. Nevertheless, there is a convolution product which turns $C_{c}(H)$ into an algebra. Thus, we drop the continuity of the product but we introduce the axioms which make the construction of the $*$-algebra $C_{c}(H)$ and its $C^{*}$-completions possible. Axioms (i), (iii), (iv), (v) directly generalize those of [2]. Axiom (ii) expresses the associativity of the product, when it is extended to bounded Radon measures. Axiom (vi) does not appear explicitly in the usual axioms for hypergroups but is a consequence of these axioms [12, Lemma 4.3.B]. The main reason to introduce this axiom is that it allows the construction of approximate units in the convolution algebra $C_{c}(H)$ just as in [21, Proposition 2.1.9]. It also implies half of the axiom (v), namely if the support of $m(x, y)$ meets $i\left(H^{(0)}\right)$, then $x=y^{*}$. Axiom (vii) means essentially separate continuity of the product.

Let us give the axioms about Haar systems.

Definition 4.3. A Haar system on a locally compact hypergroupoid $H$ is a system of Radon measures $\lambda=\left(\lambda^{u}\right)_{u \in H^{(0)}}$ for the range map such that

(i) for all $f \in C_{c}(H), u \in H^{(0)} \mapsto \int f d \lambda^{u}$ is continuous;

(ii) for all $f, g \in C_{c}(H)$ and all $x \in H$,

$$
\int f(x * y) g(y) d \lambda^{s(x)}(y)=\int f(y) g\left(x^{*} * y\right) d \lambda^{r(x)}(y)
$$

(iii) for all $f, g \in C_{c}(H), x \in H \mapsto \int f(x * y) g(y) d \lambda^{s(x)}(y)$ is continuous with compact support.

Remark 4.4. Assumption (ii) is called the adjoint property; it is formally stronger than the usual left invariance property

$$
\int f(x * y) d \lambda^{s(x)}(y)=\int f(y) d \lambda^{r(x)}(y) .
$$

It is shown to be equivalent to the left invariance property in the case of hypergroups. We expect that this equivalence still holds for our hypergroupoids; for our purpose, which is to define a *algebra structure on $C_{c}(H)$, we prefer to require the adjoint property. Assumption (iii) is also designed to turn $C_{c}(H)$ into an algebra under convolution.

Of course, our definition includes locally compact hypergroups which have a Haar measure (this includes abelian, compact or discrete hypergroups, the general case is not settled) but our main motivation is to give a framework to the spatial hypergroupoids described in the next theorem. Spatial hypergroupoids and their $C^{*}$-algebras appear in [11] but without the formal definition of a hypergroupoid. 
Theorem 4.5. Let $G$ be a locally compact groupoid with Haar system and $X$ a proper $G$ space $X$ endowed with a continuous $G$-equivariant system of measures $\alpha=\left(\alpha^{u}\right)_{u \in G^{(0)}}$. Then $H=(X * X) / G$ is a locally compact hypergroupoid with Haar system.

Proof. We assume that $X$ is a left $G$-space and denote by $r: X \rightarrow G^{(0)}$ its moment map. Recall that $G$ acts on $X * X$ by the diagonal action $\gamma(x, y)=(\gamma x, \gamma y)$. The image of $x \in X$ in $X / G$ is denoted here by $[x]$. Similarly, the image of $(x, y) \in X * X$ in $(X * X) / G$ is denoted by $[x, y]$. The unit space of $H$ is $H^{(0)}=X / G$. The range and source maps are respectively $r([x, y])=[x]$ and $s([x, y])=[y]$. The identification map $i: H^{(0)} \rightarrow H$ is $i([x])=[x, x]$. The involution map is $[x, y]^{*}=[y, x]$. The map $X * X * X \rightarrow H^{(2)}$ sending $(x, y, z)$ to $([x, y],[y, z])$ is surjective and we shall write $[x, y, z]$ instead of $([x, y],[y, z])$ the elements of $H^{(2)}$. Note that $\left[x^{\prime}, y^{\prime}, z^{\prime}\right]=[x, y, z]$ if and only there exist $(\gamma, \zeta) \in G * G(y)$ such that $x^{\prime}=\gamma x, y^{\prime}=\gamma y$ and $z^{\prime}=\gamma \zeta z$ where $G(y)=\{\zeta \in G: \zeta y=y\}$ is the isotropy group at $y$. We denote by $\beta_{y}$ the normalized Haar measure of the compact group $G(y)$ and, for $[x, y, z] \in H^{(2)}$, by $m_{[x, y, z]}$ the measure on $H$ defined by

$$
\int f d m_{[x, y, z]}=\int f[\zeta x, z] d \beta_{y}(\zeta)
$$

It is then a tedious but straightforward task to check that all the axioms of Definition 4.1 are satisfied. Here is a sketch of the proof of (vi). One introduces a proper metric $d$ on $X$ defining its topology. If $f \in C_{c}(H)$, for a fixed $y \in X$, the function $x \mapsto f[x, y]$ is uniformly continuous on $X^{r(y)}$. A compactness argument gives $\eta>0$ such that for all $(x, z) \in X * X$ such that $d(x, z)<\eta$, the inequality $|f[x, y]-f[z, y]|<\epsilon$ holds for all $y \in X$ such that $r(y)=r(x)$. The desired set $U$ is the image of the subset of $X * X$ defined by $d(x, z)<\eta$. The system of measures $\alpha$ defines a system of measures $\lambda$ for the range map $H \rightarrow X / G$ : for $x \in X, \lambda^{[x]}$ is the image of $\alpha^{r(x)}$ by the proper surjective map $\varphi^{x}: X^{r(x)} \rightarrow H^{[x]}$ sending $y$ to $[x, y]$ : for $f \in C_{c}(H)$,

$$
\int f d \lambda^{[x]}=\int f([x, y]) d \alpha^{r(x)}(y) .
$$

One checks that this depends only on $[x]$. The continuity of $\alpha$ ensures the continuity of $\lambda$. The disintegration of the measure $\alpha^{r(x)}$ along $\varphi^{x}: X^{r(x)} \rightarrow H^{[x]}$ is given by

$$
\alpha^{r(x)}=\int \beta_{[x, y]}^{x} d \lambda^{[x]}([x, y]), \quad \text { where for } \quad g \in C_{c}\left(X^{r(x)}\right) \quad \int g d \beta_{[x, y]}^{x}=\int g(\zeta y) d \beta_{x}(\zeta) .
$$

One deduces the expected expression of the convolution product: for $f, g \in C_{c}(H)$, one has

$$
\begin{aligned}
f * g[x, z] & =\int f[x, y]\left(\int g d m_{[y, x, z]}\right) d \lambda^{[x]}([x, y]) \\
& =\int f[x, y]\left(\int g[\zeta y, z] d \beta_{x}(\zeta)\right) d \lambda^{[x]}([x, y]) \\
& =\int\left(\int f\left[x, \cdot g[\cdot, z] d \beta_{[x, y]}^{x}\right) d \lambda^{[x]}([x, y])=\int f[x, y] g[y, z] d \alpha^{r(x)}(y) .\right.
\end{aligned}
$$

From this expression of the convolution product, one can see that the condition (iii) of Definition 4.3 is satisfied. The adjoint property (ii) is also easily checked.

Let us formalize a definition used earlier.

Definition 4.6. A locally compact hypergroupoid with Haar system of the form $(X * X) / G$ given by the theorem, where $G$ is a locally compact groupoid with Haar system and $X$ is a proper $G$-space endowed with a continuous $G$-equivariant system of measures is called a spatial hypergroupoid. 
Given a locally compact hypergroupoid with Haar system $(H, \lambda)$, we define the convolution product of $f$ and $g$ in $C_{c}(H)$ by

$$
f * g(x)=\int f(x * y) g\left(y^{*}\right) d \lambda^{s(x)}(y)=\int f(y) g\left(y^{*} * x\right) d \lambda^{r(x)}(y)
$$

and the involution by $f^{*}(x)=\overline{f\left(x^{*}\right)}$.

Proposition 4.7. Let $(H, \lambda)$ be a locally compact hypergroupoid with Haar system. Endowed with the convolution product, the involution and the inductive limit topology, $C_{c}(H)$ is a topological *-algebra.

The proof is very much like the groupoid case (see [21, Proposition II.1.1]) and will not be given here.

The definition of the full $C^{*}$-algebra $C^{*}(H, \lambda)$ and the reduced $C^{*}$-algebra $C_{r}^{*}(G, \lambda)$ follows the same line as the groupoid case.

Definition 4.8. The full $C^{*}$-algebra $C^{*}(H, \lambda)$ of a locally compact hypergroupoid with Haar system $(H, \lambda)$ is the completion of the $*$-algebra $C_{c}(H)$ with respect to the full norm

$$
\|f\|=\sup \left\{\|L(f)\|: L \text { non-degenerate and I-bounded representation of } C_{c}(H)\right\},
$$

where the I-norm of $f \in C_{c}(H)$ is

$$
\|f\|_{I}=\max \left(\sup _{u \in H^{(0)}} \int|f| d \lambda^{u}, \sup _{u \in H^{(0)}} \int\left|f^{*}\right| d \lambda^{u}\right) .
$$

In the case of a hypergroup (i.e. $H^{(0)}$ has only one element), this definition agrees with that given in $[7,8]$, except that we define the involution without using the modular function.

Definition 4.9. The reduced $C^{*}$-algebra $C_{r}^{*}(H, \lambda)$ of a locally compact hypergroupoid with Haar system $(H, \lambda)$ is the completion of $C_{c}(H)$ for the reduced norm

$$
\|f\|_{r}=\sup \left\{\left\|L_{u}(f)\right\|: u \in H^{(0)}\right\}
$$

where $L_{u}$ is the $*$-representation of the $*$-algebra $C_{c}(H)$ on the Hilbert space $L^{2}\left(H_{u}, \lambda_{u}\right)$ defined by $L_{u}(f) \xi(x)=\int f(x * y) \xi\left(y^{*}\right) d \lambda^{u}(y)$ for $f \in C_{c}(H)$ and $\xi \in C_{c}\left(H_{u}\right)$.

When $H=(X * X) / G$ is a spatial hypergroupoid, where $G$ is a locally compact groupoid with Haar system $\lambda$ and $X$ is a proper $G$-space with equivariant sytem $\alpha$, we have defined the $C^{*}$ algebra $C_{G}^{*}(H)$ by considering only the representations of $C_{c}(H)$ induced by representations of $G$. Since they are I-bounded, $C_{G}^{*}(H)$ is a quotient of $C^{*}(H)$. The examples of [12, Subsection 15.5] or [8, Section 4]) show that $C_{G}^{*}(H)$ may be a strict quotient of $C^{*}(H)$. On the other hand, the completion of $C_{c}(H)$ obtained from the regular representation of $G$ agrees with the reduced $C^{*}$-algebra $C_{r}^{*}(H)$.

\section{Acknowledgements}

I thank S. Echterhoff for the references [7, 8] and J. Brown, R.D. Holkar, G. Nagy and S. Reznikoff for fruitful discussions. I thank the referees for their great help to improve the presentation of the manuscript. 


\section{References}

[1] Anantharaman-Delaroche C., Renault J., Amenable groupoids, Monographies de L'Enseignement Mathématique, Vol. 36, L'Enseignement Mathématique, Geneva, 2000.

[2] Bloom W.R., Heyer H., Harmonic analysis of probability measures on hypergroups, de Gruyter Studies in Mathematics, Vol. 20, Walter de Gruyter \& Co., Berlin, 1995.

[3] Bourbaki N., Éléments de mathématique. Fascicule XXIX. Livre VI: Intégration. Chapitre 7: Mesure de Haar. Chapitre 8: Convolution et représentations, Actualités Scientifiques et Industrielles, No. 1306, Hermann, Paris, 1963.

[4] Buneci M., Stachura P., Morphisms of locally compact groupoids endowed with Haar systems, math.OA/0511613.

[5] Dunkl C.F., The measure algebra of a locally compact hypergroup, Trans. Amer. Math. Soc. 179 (1973), $331-348$.

[6] Hauenschild W., Kaniuth E., Kumar A., Harmonic analysis on central hypergroups and induced representations, Pacific J. Math. 110 (1984), 83-112.

[7] Hermann P., Induced representations of hypergroups, Math. Z. 211 (1992), 687-699.

[8] Hermann P., Representations of double coset hypergroups and induced representations, Manuscripta Math. 88 (1995), 1-24.

[9] Hilsum M., Skandalis G., Morphismes $K$-orientés d'espaces de feuilles et fonctorialité en théorie de Kasparov (d'après une conjecture d'A. Connes), Ann. Sci. École Norm. Sup. (4) 20 (1987), 325-390.

[10] Holkar R.D., Topological construction of $C^{*}$-correspondences for groupoid $C^{*}$-algebras, Ph.D. thesis, Göttingen University, in preparation.

[11] Holkar R.D., Renault J., Hypergroupoids and $C^{*}$-algebras, C. R. Math. Acad. Sci. Paris 351 (2013), 911914, arXiv:1403.3424.

[12] Jewett R.I., Spaces with an abstract convolution of measures, Adv. Math. 18 (1975), 1-101.

[13] Kalyuzhnyi A.A., Podkolzin G.B., Chapovsky Yu.A., Harmonic analysis on a locally compact hypergroup, Methods Funct. Anal. Topology 16 (2010), 304-332.

[14] Landsman N.P., Operator algebras and Poisson manifolds associated to groupoids, Comm. Math. Phys. 222 (2001), 97-116.

[15] Macho Stadler M., O’uchi M., Correspondence of groupoid $C^{*}$-algebras, J. Operator Theory 42 (1999), 103-119.

[16] Mackey G.W., Induced representations of locally compact groups. I, Ann. of Math. 55 (1952), 101-139.

[17] Mrčun J., Functoriality of the bimodule associated to a Hilsum-Skandalis map, K-Theory 18 (1999), 235253.

[18] Muhly P.S., Renault J., Williams D.P., Equivalence and isomorphism for groupoid $C^{*}$-algebras, J. Operator Theory 17 (1987), 3-22.

[19] Ramsay A., Topologies on measured groupoids, J. Funct. Anal. 47 (1982), 314-343.

[20] Ramsay A., The Mackey-Glimm dichotomy for foliations and other Polish groupoids, J. Funct. Anal. 94 (1990), 358-374.

[21] Renault J., A groupoid approach to $C^{*}$-algebras, Lecture Notes in Mathematics, Vol. 793, Springer, Berlin, 1980.

[22] Renault J., Strong Morita equivalence of groupoid $C^{*}$-algebras, Talk given in the Operator Algebras Section of the Annual Summer Meeting of the Canadian Mathematical Society (Halifax, May 27-30, 1981).

[23] Renault J., $C^{*}$-algebras of groupoids and foliations, in Operator Algebras and Applications, Part I (Kingston, Ont., 1980), Proc. Sympos. Pure Math., Vol. 38, Amer. Math. Soc., Providence, R.I., 1982, 339-350.

[24] Renault J., Représentation des produits croisés d'algèbres de groupoïdes, J. Operator Theory 18 (1987), 67-97.

[25] Renault J., The ideal structure of groupoid crossed product $C^{*}$-algebras, J. Operator Theory 25 (1991), $3-36$. 
[26] Rieffel M.A., Induced representations of $C^{*}$-algebras, Adv. Math. 13 (1974), 176-257.

[27] Rieffel M.A., Applications of strong Morita equivalence to transformation group $C^{*}$-algebras, in Operator Algebras and Applications, Part I (Kingston, Ont., 1980), Proc. Sympos. Pure Math., Vol. 38, Amer. Math. Soc., Providence, R.I., 1982, 299-310.

[28] Spector R., Aperçu de la théorie des hypergroupes, in Analyse harmonique sur les groupes de Lie (Sém. Nancy-Strasbourg, 1973-75), Lecture Notes in Math., Vol. 497, Springer, Berlin, 1975, 643-673.

[29] Tu J.-L., Non-Hausdorff groupoids, proper actions and K-theory, Doc. Math. 9 (2004), 565-597, math.OA/0403071. 